\section{Schweizerische Gesellschaft für} Anästhesiologie und Reanimation

\section{Société Suisse d'Anesthésiologie} et de Réhabilitation

Vorstand/Comité

Präsidentin:

PD Dr Elisabeth Van Gessel, Genève

Past-President:

Prof. Dr. Franz Frei, Basel

Mitglieder:

PD Dr. med. Karl Friederich Hampl, Aarau; Dr. med. Christof Heim, Münsterlingen; Prof. Dr. med. Thomas Pasch, Zürich; Prof. Dr Patrick Ravussin, Sion;

Dr Max Wintsch, Yverdon-les-Bains

Sekretariat:

SGAR, Postfach, 3000 Bern 25, Tel. 03133234 33, Fax 03133298 79, E-mail: bbscongress@swissonline.ch

\section{Schweizerische Ärztegesellschaft für Manuelle Medizin (SAMM)}

Vorstand

\section{Präsident:}

Marc-Henri Gauchat, Sion

\section{Mitglieder:}

Ueli Affolter, Weier im Emmental;

Marcus R. Baumann, Basel;

Hans-Rudolf Beusch, Spiez;

Ulrich W. Böhni, Schaffhausen;

Jiri Dvorak, Zürich;

Balz Kleinert, Zürich;

Jean-Dominique Lavanchy, Yvonand;

Daniel Loustalot, Locarno;

Bernard Terrier, Baden

Sekretariat:

SAMM-Sekretariat,

Picassoplatz 4, 4052 Basel,

Tel. 06127991 40, Fax 06127893 96,

E-Mail: info@samm.ch,

Website: www.samm.ch

Vertreter der Schweiz in der FIMM

Präsident:

Bernard Terrier, Baden

Kassier:

Vaclav Dvorak, Bonaduz

Educational Committee:

Marc-Henri Gauchat, Sion

\section{Schweizerische Gesellschaft für Nephrologie}

Vorstand

Präsident:

H. Murer, Zürich

President-elect:

H. P. Marti, Bern

Sekretär:

P. Sandoz, Solothurn

Kassier:

G. Vogel, Monthey

Beisitzer:

J. P. Guignard, Lausanne

Beirat

P. Ambühl, Zürich; A. Bock, Aarau;

F. Frey, Bern; E. Girardin, Genève;

H. Heule, Altstätten; P. Y. Martin, Genève;

M. Mihatsch, Basel; D. Robert, Neuchâtel;

B. Rossier, Lausanne; R. Wüthrich, St. Gallen

\section{Schweizerische Ärztegesellschaft für Manuelle Medizin (SAMM)}

Schlussprüfung zur Erlangung des Fähigkeitsausweises Manuelle Medizin (SAMM) und der Mitgliedschaft SAMM

Die Schweizerische Ärztegesellschaft für Manuelle Medizin (SAMM) führt im November 2003 die Schlussprüfungen zur Erlangung des Fähigkeitsausweises für Manuelle Medizin (SAMM) bzw. der Mitgliedschaft SAMM durch.

Ort und Zeitpunkt

Schlussprüfung deutsch/französisch: Kantonsspital Fribourg, Donnerstag, 6. November 2003; Schlussprüfungen deutsch: Schulthess-Klinik Zürich, Donnerstag, 13. November 2003

Anmeldungen bis 1. Oktober 2003 an: Sekretariat SAMM, Picassoplatz 4, 4052 Basel, Tel. 06127991 40, Fax 0612789396

Prüfungsgebühr: Die SAMM erhebt einen Unkostenbeitrag von Fr. 235.-.

\section{Société médicale suisse de médecine manuelle (SAMM)}

Examen final pour l'obtention de l'Attestation de formation complémentaire en médecine manuelle (SAMM) et de l'affiliation à la SAMM

La Société médicale suisse de médecine manuelle organise en novembre 2003 l'examen final pour l'obtention de l'attestation de formation complémentaire en médecine manuelle (SAMM) et de l'affiliation à la SAMM.

\section{Lieu et date}

Examen français/allemand: Hôpital cantonal de Fribourg, Jeudi, le 6 novembre 2003

Inscriptions jusqu' au $1^{\text {er }}$ octobre 2003 auprès du Secrétariat SAMM, Picassoplatz 4, 4052 Bâle, tél. 06127991 40, fax 0612789396.

Taxe d'examen: La SAMM prélève une participation aux frais de Fr. 235.-.

La réussite de l'examen intermédiaire permet de devenir membre Extraordinaire de la SAMM 


\section{Schweizerische Gesellschaft für Pneumologie}

Sachkunde für dosisintensives Röntgen in der Pneumologie: Theoretischer Kurs (StSV Art. 11)

Aufgrund des Weiterbildungsprogrammes in Pneumologie, welches am 1. Januar 2001 in Kraft gesetzt wurde, ist die Teilnahme an einem theoretischen Kurs in Sachkunde für dosisintensives Röntgen für Anwärter für den Facharzttitel für Pneumologie obligatorisch, die ihre Weiterbildung nach dem 31. Dezember 2002 abschliessen. Ein 31/2tägiger Kurs wird deshalb organisiert.

Kurs für die Ausbildung im Bereich Strahlenschutz zum Sachverständigen (StSV Art. 18)

Pneumologen, die zudem eine Bewilligung zum Betreiben einer eigenen Röntgenanlage erhalten wollen, können den anschliessend stattfindenden 1 1 $\frac{1}{2}$ tägigen Kurs zur Erlangung des Sachverstandes absolvieren.

Ort: Abteilung Radiologische Physik, Prof. Dr. J. Roth, Kantonsspital, 4031 Basel

Kursdaten: 12.-15. Mai 2003 für Sachkunde dosisintensives Röntgen 15.-16. Mai 2003 für Sachverstand

Zulassungsbedingung zu den Prüfungen: Sachkunde: theoretischer Kurs Sachkunde Sachverstand: theoretischer Kurs Sachverstand

Kursgebühr: Sachkunde: Fr. 1400.Sachverstand: Fr. 400.-

\section{Auskunft und Einschreibung:}

Frau I. Burgy-Landolf, Abteilung Radiologische Physik, Kantonsspital, 4031 Basel,

Tel. 06126526531 40, Fax 0612653135 , E-Mail: iburgy@uhbs.ch

Zertifikat: Abteilung Radiologische Physik (Herausgeber)

Einschreibefrist: 15. Februar 2003

\section{Société Suisse de Pneumologie}

Qualification pour les examens radiologiques à fortes doses en pneumologie: cours théorique (ORaP, art. 11)

Conformément au programme de formation postgraduée en pneumologie, en vigueur depuis le $1^{\text {er }}$ janvier 2001, la participation à un cours théorique de qualification pour les examens radiologiques à fortes doses est obligatoire pour les pneumologues terminant leur formation postgraduée après le 31 décembre 2002. Ce cours durera $3 \frac{1}{2}$ jours.

Cours de formation en radioprotection (ORaP, art. 18)

Les futurs spécialistes en pneumologie désirant obtenir une autorisation pour l'exploitation d'une installation à rayons $\mathrm{X}$ à usage médical pourront suivre un complément de cours d'une durée de $1 \frac{1}{2}$ jours.

Organisation: Fondation PRORAME, Secrétariat, Institut de radiophysique appliquée, Prof. Dr Jean-François Valley, 1, Grand-Pré, 1007 Lausanne

Date: 16 au 20 juin 2003

Durée: Qualification: lundi au jeudi midi (31/2 jours)

Radioprotection: jeudi midi au vendredi ( $1 \frac{1}{2}$ jours)

Conditions d'admission pour les examens: Qualification: cours théorique qualification Radioprotection: cours théorique radioprotection

Taxe du cours: Qualification: Fr. 1400.Radioprotection: Fr. 400.-

Renseignements/Inscriptions:

Prof. Dr Jean-François Valley, tél. 02162334 40, fax 02162334 35, e-mail: jean-francois.valley@inst.hospvd.ch

Certificat: Fondation PRORAME

Délai d'inscription: 15 février 2003

\section{SGI-Preis 2003}

Im Rahmen der Jahrestagung der Schweizerischen Gesellschaft für Intensivmedizin ist für das Jahr 2003 ein Preis von Fr. 8000.- für die beste Originalarbeit auf dem Gebiet der Intensivmedizin ausgeschrieben.

Die Arbeiten sollten aus einer schweizerischen Intensivstation stammen und in den Jahren 2002 und 2003 publiziert oder zur Publikation in einer Fachzeitschrift angenommen worden sein.

Die Manuskripte müssen bis allerspätestens am 15. Februar 2003 an den Präsidenten der SGI, PD Dr. Reto Stocker, Abteilung Chirurgische Intensivmedizin, Universitätsspital Zürich, Rämistrasse 100, 8091 Zürich, eingesandt werden.

Die Preisverleihung findet anlässlich der Jahresversammlung am 8./9. Mai in Lausanne statt.

\section{Prix SGI 2003}

Dans le cadre de la réunion annuelle de la Société Suisse de Médecine Intensive, un prix de fr. 8000.- sera attribué pour le meilleur travail scientifique publié dans le domaine de la médecine intensive.

Ce prix concerne les travaux publiés ou acceptés pour publication en 2002 et 2003 dans des revues révisées. Ceux-ci doivent provenir d'une unité de soins intensifs suisse. Les manuscrits sont à adresser aux PD Dr. Reto Stocker, Président SSMI, Abteilung Chirurgische Intensivmedizin, Universitätsspital Zürich, Rämistrasse 100, 8091 Zurich, au plus tard jusqu'au 15 février 2003.

Le prix sera remis au bénéficiaire lors de la réunion annuelle 8/9 mai 2003 à Lausanne. 


\section{Preis der Schweizerischen Gesellschaft für Thorax-, Herz- und Gefässchirurgie (SGTHGC)}

Die Schweizerische Gesellschaft für ThoraxHerz- und Gefässchirurgie verleiht jährlich eine mit Fr. 10000.- dotierte Auszeichnung für eine hervorragende wissenschaftliche Arbeit aus dem Gebiet der Thorax-, Herz- und Gefässchirurgie. Die vorgelegte Arbeit darf nicht für einen anderen Preis eingereicht worden sein. Sie kann als Manuskript oder als Sonderdruck vorgelegt werden. Im Falle einer bereits erfolgten Veröffentlichung darf diese nicht länger als 6 Monate zurückliegen.

Bewerber reichen ihre Arbeit in 4 facher Ausführung bis am 15. Februar 2003 dem Sekretär der Schweizerischen Gesellschaft für Thorax-, Herz- und Gefässchirurgie ein.

Die Preisverleihung findet anlässlich des Jahreskongresses der Schweizerischen Gesellschaft für Kardiologie, der Schweizerischen Gesellschaft für Intensivmedizin und der Schweizerischen Gesellschaft für Thorax-, Herz- und Gefässchirurgie in Lausanne (8.-10. Mai 2003) statt.

Prof. T. Carrel, Sekretär der SGTHGC, Klinik für Herz- und Gefässchirurgie, Inselspital, 3010 Bern.

\section{Prix de la Société suisse de chirurgie thoracique et cardio- vasculaire (SSCTCV)}

La Société suisse de chirurgie thoracique et cardio-vasculaire attribue un prix de Fr. 10 000.- destiné à récompenser un travail scientifique exceptionnel dans le domaine de la chirurgie thoracique et cardio-vasculaire. Le travail ne doit pas avoir été soumis pour un autre prix. Il peut s'agir d'un manuscrit ou d'un tiré à part; en cas de publication, cette dernière ne doit pas avoir dépassé un délai de 6 mois.

Les candidats sont invités à adresser leur texte avant le 15 février 2003 en 4 exemplaires au secrétaire de la Société suisse de chirurgie thoracique et cardio-vasculaire.

Le prix sera remis lors du congrès annuel de la Société suisse de cardiologie, de la Société suisse de médecine intensive et de la Société suisse de chirurgie thoracique et cardio-vasculaire à Lausanne (8 au 10 mai 2003).

Prof. T. Carrel, secrétaire de la SSCTCV, Service de chirurgie cardio-vasculaire, Hôpital de l'Ile, 3010 Berne.

\section{Maximilian-Forschungsförderpreis HERZKIND e.V. 2003}

Die Eltern- und Patientenselbsthilfeorganisation HERZKIND e.V. vergibt 2003 erneut einen mit maximal 25000 Euro dotierten Preis zur Förderung der wissenschaftlichen Forschung auf dem Gebiet der Pädiatrischen Kardiologie. Der Förderpreis kann an europäische Institutionen vergeben werden, die ein wissenschaftliches Projekt im Bereich der Grundlagenforschung, der klinischen Diagnostik, der kardiologischen bzw. chirurgischen Interventionen sowie der Langzeitbetreuung durchzuführen beabsichtigen. Vorhaben, die eine Brücke zwischen Grundlagen- und klinischer Forschung bilden, werden bevorzugt berücksichtigt.

Das Preisgeld soll im wesentlichen der Deckung oder Ergänzung von Personal- und oder Sachmitteln, nicht jedoch der Gerätebeschaffung dienen. Bewerbungen mit detaillierter Darstellung des wissenschaftlichen Projekts, des Zeitplans und der Finanzierung zusammen mit Lebenslauf und wissenschaftlichem Werdegang des Hauptantragstellers sowie Angaben zu eigenen Vorarbeiten auf dem Forschungsgebiet werden in vierfacher Ausfertigung erbeten bis zum 31. Mai 2003 an den Vorstand des HERZKIND e.V., Frau Hogendoorn, Husarenstrasse 70, D-38102 Braunschweig.

\section{Neue Broschüre «Wohnen und Gesundheit»}

Mit dieser neuen Broschüre der «Ärztinnen und Ärzte für Umweltschutz» werden der Ärzteschaft, den Interessierten und den Betroffenen von Wohnraumproblemen gesundheitsrelevante Informationen über Innenraum bzw. das Wohnumfeld zur Verfügung gestellt. Der Inhalt behandelt den derzeitigen Wissensstand von Umweltpsychosomatik, Allergien, chemische und mikrobielle Verunreinigungen in Innenräumen sowie die Radonproblematik und elektromagnetische Felder im Wohnbereich. Zahlreiche praktische Hinweise zu Ursachen, Erkrankungen, möglichen Abklärungs- und Behandlungswegen, immer mit Bezug auf die eigene Wohnung, sind verständlich dargelegt.

In der Schweiz verbringen die Menschen den grössten Teil ihrer Arbeits- und Freizeit in geschlossenen Räumen. Sie werden so vom Raumklima sehr stark beeinflusst. Wohlbefinden und Gesundheit sind von der Gestaltung des Arbeits- und Wohnumfeldes, den verwendeten Materialien, der Temperatur, der Luftzirkulation und der Luftqualität im Innenraum abhängig. Bei ursächlich unklaren Krankheiten sollte deshalb immer auch an die Möglichkeit von Innenraumbelastungen gedacht und entsprechend gehandelt werden. Belastende Substanzen aus Baustoffen, Einrichtungsgegenständen und Haushaltprodukten, ungenügende Lüftung und Feuchtigkeitsprobleme, die das Wohnklima belasten, können teilweise zu schweren Gesundheitsbeeinträchtigungen führen. Die Broschüre will darauf aufmerksam machen mit einer Fülle von Beispielen und interessantem Hintergrundwissen. Wichtige Adressen runden die Informationen ab.

Die Broschüre «Wohnen und Gesundheit» ist zum Preis von Fr. 10.- plus Porto zu beziehen bei: Ärztinnen und Ärzte für Umweltschutz, Postfach 11, 4013 Basel, Tel. 06132249 49, Fax 06132248 51, E-Mail: info@aefu.ch.

Ärztinnen und Ärzte für Umweltschutz 